\title{
Development of a Broad-Range 23S rDNA Real-Time PCR Assay for the Detection and Quantification of Pathogenic Bacteria in Human Whole Blood and Plasma Specimens
}

\author{
Paolo Gaibani, ${ }^{1,2}$ Mara Mariconti, ${ }^{3,4}$ Gloria Bua, ${ }^{1}$ Sonia Bonora, \\ Davide Sassera, ${ }^{3}$ Maria Paola Landini, ${ }^{1}$ Patrizia Mulatto, ${ }^{4}$ Stefano Novati, ${ }^{4}$ \\ Claudio Bandi, ${ }^{3}$ and Vittorio Sambri ${ }^{1}$ \\ ${ }^{1}$ Operative Unit of Clinical Microbiology, St. Orsola-Malpighi University Hospital, 40138 Bologna, Italy \\ ${ }^{2}$ Department of Haematology and Oncology "L. and A. Seragnoli", Unit of Clinical Microbiology, Regional Reference \\ Centre for Microbiological Emergencies (CRREM), St. Orsola-Malpighi Hospital, University of Bologna, 9 Via G. Massarenti, \\ 40138 Bologna, Italy \\ ${ }^{3}$ DIVET, University of Milan, 20100 Milan, Italy \\ ${ }^{4}$ Fondazione IRCCS Policlinico San Matteo, 27100 Pavia, Italy
}

Correspondence should be addressed to Paolo Gaibani; paolo.gaibani@unibo.it

Received 17 October 2012; Revised 15 January 2013; Accepted 29 January 2013

Academic Editor: Arun K. Bhunia

Copyright (C) 2013 Paolo Gaibani et al. This is an open access article distributed under the Creative Commons Attribution License, which permits unrestricted use, distribution, and reproduction in any medium, provided the original work is properly cited.

\begin{abstract}
Molecular methods are important tools in the diagnosis of bloodstream bacterial infections, in particular in patients treated with antimicrobial therapy, due to their quick turn-around time. Here we describe a new broad-range real-time PCR targeting the 23S rDNA gene and capable to detect as low as 10 plasmid copies per reaction of targeted bacterial 23S rDNA gene. Two commercially available DNA extraction kits were evaluated to assess their efficiency for the extraction of plasma and whole blood samples spiked with different amount of either Staphylococcus aureus or Escherichia coli, in order to find the optimal extraction method to be used. Manual QIAmp extraction method with enzyme pre-treatment resulted the most sensitive for detection of bacterial load. Sensitivity of this novel assay ranged between 10 and $10^{3}$ CFU per PCR reaction for E. coli and S. aureus in human whole blood samples depending on the extraction methods used. Analysis of plasma samples showed a 10- to 100 -fold reduction of bacterial $23 \mathrm{~S}$ rDNA in comparison to the corresponding whole blood specimens, thus indicating that whole blood is the preferential sample type to be used in this real-time PCR protocol. Our results thus show that the $23 \mathrm{~S}$ rDNA gene represents an optimal target for bacteria quantification in human whole blood.
\end{abstract}

\section{Introduction}

Blood culture (BC) is the most widely used method for the diagnosis of bloodstream bacterial infections (BSIs) [1]. Major limitations of culture techniques are the intrinsic poor cultivability (or noncultivability) of some bacteria and the inhibitory effects of concurrent antibiotic therapy. In addition, the turn-around time of $\mathrm{BC}$ ranges from 24 to 72 hours, which implies that results might become available too late to be of clinical utility [2]. In recent years, molecular methods have been proposed as additional diagnostic tools for BSIs [2, 3]. Several studies reported the development and clinical assessment of broad-range real-time PCR protocols, capable of rapid detection and identification of a vast proportion of cultivable and uncultivable bacteria, from different types of biological samples [4-12]. The majority of the broad-range real-time PCRs use a single pair of universal primers to identify bacteria through the amplification of the 16S ribosomal DNA (16S 0072DNA), given the high level of homology of this gene throughout the bacterial species diversity [12]. The amplification of the $16 \mathrm{~S}$ rDNA has been described as a specific and sensitive tool to identify and quantify different microorganisms depending on the specific protocol used [7, 13-17]. A major pitfall of 16S-based 
panbacterial primers is their cross-reactivity with human ribosomal RNA genes; to overcome the problem, Kommedal and coworkers proposed a $16 \mathrm{~S}$ rDNA-based dual-priming protocol [18]. In addition to $16 \mathrm{~S}$ rDNA, the gene coding for the large subunit ribosomal RNA (23S rDNA) has also been targeted for the development of PCR methods for bacterial detection, but only a limited number of studies evaluated the utility of 23S-based panbacterial primers [4, 19], and no studies have so far exploited this target for BSI monitoring. The aim of our study was to develop a novel 23S rDNAtargeted real-time pan-bacterial PCR method, suitable for the detection of a wide range of bacterial species, for the monitoring of BSIs. In addition, since the amount of bacterial DNA detected in blood from healthy subjects is reported to be highly variable and profoundly influenced by the use of whole blood or plasma $[4,7,20]$, we tested the suitability of different extraction procedures for the isolation of bacterial DNA from blood-derived specimens.

\section{Materials and Methods}

2.1. Design of the $23 S$ rDNA Universal Primers. Complete $23 \mathrm{~S}$ rDNA sequences from 50 bacterial species, spanning the eubacterial diversity, were retrieved from the NCBI database (http://www.ncbi.nlm.nih.gov/). Alignment of the sequences was performed using the MUSCLE software [21] and manually checked. 28S rDNA sequences from Caenorhabditis elegans, Candida albicans, Drosophila melanogaster, and Homo sapiens were also included in the alignment, in order to evaluate the specificity for the bacterial rDNA of the designed primers. Primers were manually designed on the obtained alignment, and then evaluated using mfold (http://mfold.rna.albany.edu/?q=mfold) and the Operon oligo analysis tool (http://www.operon.com/tools/ oligo-analysis-tool.aspx). The sequences of the $23 \mathrm{~S}$ rDNAtargeted pan-bacterial primers are PAN23S-F, $5^{\prime}$-TCGCTCAACGGATAAAAG-3 ${ }^{\prime}$ and PAN23S-R, $5^{\prime}$-GATGAnCCGACATCGAGGTGC- $3^{\prime}$; the amplified fragment size is 97 base pairs in Escherichia coli. The designed primers were then compared to the nonredundant nucleotide eukaryotic database using the Blast software (http://blast.ncbi.nlm.nih .gov/Blast.cgi), to highlight possible unwanted matches.

2.2. $23 S$ rDNA Real-Time PCR. PCR reactions were effected in a final volume of $25 \mu \mathrm{L}$, containing $12.5 \mu \mathrm{L}$ of SYBR Green PCR Master Mix Reagent (Biorad-Hercules, CA, USA), $250 \mathrm{nM}$ of each primer, and $5 \mu \mathrm{L}$ of the extracted DNA solution. PCR was performed in an IQ5 thermocycler (Biorad-Hercules, CA, USA) with an initial step of $5 \mathrm{~min}$ at $95^{\circ} \mathrm{C}$, followed by 40 cycles of $15 \mathrm{~s}$ at $95^{\circ} \mathrm{C}$ and $30 \mathrm{~s}$ at $58^{\circ} \mathrm{C}$. After PCR amplification, the melting curve was established by increasing the temperature from $55^{\circ} \mathrm{C}$ to $95^{\circ} \mathrm{C}$.

2.3. Bacterial Isolates. A panel of 47 different bacterial isolates, 20 Gram-positive, and 27 Gram-negative was included in the study (Table 1). These strains were either obtained from routine cultures, identified at the Unit of Clinical Microbiology, St. Orsola Malpighi Hospital, or obtained from
TABLE 1: Bacterial strains utilized in this study. The strains were either obtained from the bacterial collection of the St. Orsola Hospital (BACSO) or derived from routine workflow. In this last case the procedure for identification are reported in the Qualty Assurance files of the Laboratory.

\begin{tabular}{|c|c|}
\hline Microorganisms species & Origin of the isolate \\
\hline Acinetobacter baumannii & Urine \\
\hline Acinetobacter lwoffii & Urine \\
\hline Alcaligenes xylosoxidans & Urine \\
\hline Bacteroides fragilis & Cerebrospinal fluid \\
\hline Campylobacter jejuni & Feces \\
\hline Citrobacter braakii & Abdominal drainage \\
\hline Citrobacter freundii & Blood \\
\hline Citrobacter koseri & Urine \\
\hline Corynebacterium jeikeium & Blood \\
\hline Corynebacterium minutissimum & Blood \\
\hline Corynebacterium striatum & Blood \\
\hline Corynebacterium urealyticum & Blood \\
\hline Enterobacter cloacae & Urine \\
\hline Enterobacter aerogene & Urine \\
\hline Enterococcus casseliflavus & Blood \\
\hline Enterococcus faecalis & Bacso/atcc 29212 \\
\hline Enterococcus faecium & Blood \\
\hline Enterococcus gallina rum & Blood \\
\hline Escherichia coli & Bacso/atcc 25922 \\
\hline Haemophilus influenzae & Bacso/atcc 49247 \\
\hline Haemophilus influenzae & Bacso/atcc 49766 \\
\hline Hafnia alvei & Bile \\
\hline Klebsiella oxytoca & Blood \\
\hline Klebsiella pneumoniae & Urine \\
\hline Morganella morganii & Urine \\
\hline Nocardia sp. & Bronchial aspirate \\
\hline Proteus mirabilis & Urine \\
\hline Proteus vulgaris & Bronchial aspirate \\
\hline Providencia stuartii & Urine \\
\hline Pseudomonas aeruginosa & Bacso/atcc 27853 \\
\hline Pseudomonas luteola & Bronchial aspirate \\
\hline Salmonella sp. Group B & Feces \\
\hline Salmonella sp. Group C & Feces \\
\hline Salmonella sp. Group D & Feces \\
\hline Serratia marcescens & Urine \\
\hline Staphylococcus aureus & Bacsoatcc 29213 \\
\hline Staphylococcus epidermidis & Blood \\
\hline Staphylococcus haemolyticus & Blood \\
\hline Staphylococcus hominis & Blood \\
\hline Staphylococcus warneri & Blood \\
\hline Stenotrophomonas maltophilia & Faringeal swab \\
\hline Streptococcus agalactiae & Uretral swab \\
\hline Streptococcus anginosus & Blood \\
\hline Streptococcus mitis & Blood \\
\hline Streptococcus parasanguinis & Blood \\
\hline Streptococcus pyogene & Faringeal swab \\
\hline Streptococcus pneumoniae & Bacso/atcc 49619 \\
\hline
\end{tabular}

the bacterial collection at the same Institution (BACSO). A cell suspension containing $10^{8} \mathrm{CFU} / \mathrm{mL}$ was obtained from each bacterial isolate, and the DNA was extracted using described protocols [22]. PCR products obtained from bacterial cultures were then sequenced to verify whether just bacterial DNA had been amplified and cloned. In addition, 
five eukaryotic species from the genus Candida (Candida albicans, Candida glabrata, Candida tropicalis, Candida parapsilosis, and Candida guilliermondii) were included in the study.

2.4. PCR Sensitivity Test. An external standard for absolute quantification (i.e., the target $23 \mathrm{~S}$ rDNA gene fragment, cloned into a plasmid vector) was prepared. PCR was effected on Staphylococcus aureus DNA using the above described primers PAN23S-F and PAN23S-R according to standard PCR conditions. The band resolved on a $2 \%$ agarose gel was excised and the PCR product was then purified, quantified, and cloned using the pGEM T-easy vector (Qiagen, Basel, Switzerland) according to manufacturers' instructions. Ten randomly selected clones were sequenced with ABI technology. A plasmid containing the $23 \mathrm{~S}$ rDNA insert was purified from one of the clones, using the QIAprep Spin Miniprep Kit (Qiagen, Basel, Switzerland). After quantification, a serial dilution of the plasmid was used to assess the sensitivity of the above PCR assay, with plasmid at concentrations ranging from $10^{8}$ to $10^{1}$ copies per reaction, to generate the standard curve.

2.5. DNA Extraction from Whole Blood Spiked with GramPositive and Gram-Negative Bacteria. Tenfold serial dilution of bacteria in blood was prepared, by spiking fresh $\mathrm{K}_{3}$ EDTA blood samples with S. aureus ATCC 25923 or E. coli ATCC 25922 (as representative strains for Gram-positive and Gramnegative bacteria), to obtain final concentrations of bacteria ranging from $10^{7} \mathrm{CFU}$ per $\mathrm{mL}$ to $10 \mathrm{CFU}$ per $\mathrm{mL}$ of blood. A written informed consent was obtained from each blood donor following the ethical rules of the St. Orsola Hospital Blood Bank in Bologna. As a control, identical series were prepared in phosphate buffer saline (PBS). Standard $100 \mu \mathrm{L}$ volumes from each sample of these spiked series (blood or PBS) were subjected to DNA extraction, using the different protocols reported below. Two different commercially available methods were used following the manufacturers' instructions: the automated nucleic acid extractor NucliSens EasyMag (BioMerieux, Marcy l'Etoile, France) and the manual QIAmp DNA blood mini kit (Qiagen, Basel, Switzerland). As a third option, the following modification of the QIAmp DNA blood mini kit was also used: $100 \mu \mathrm{L}$ of fresh whole blood or PBS spiked series were preincubated with $90 \mu \mathrm{L}$ of the enzyme solution buffer $(20 \mathrm{mg} / \mathrm{mL}$ lysozyme, $20 \mathrm{mM}$ Tris $\mathrm{HCl}, 2 \mathrm{mM}$ EDTA, $1 \%$ Triton). After $2 \mathrm{~h}$ of incubation at $37^{\circ} \mathrm{C}$, the mixture was incubated at $56^{\circ} \mathrm{C}$ for $2 \mathrm{~h}$ with addition of $10 \mu \mathrm{L}$ of proteinase $\mathrm{K}$, at a concentration of $20 \mathrm{mg} / \mathrm{mL}$ (SigmaAldrich, St. Louis, MO, USA) and $100 \mu \mathrm{L}$ of AL Buffer. Then, the mixture (of $300 \mu \mathrm{L}$ ) was subjected to DNA extraction with the QIAmp DNA blood mini kit. The DNA obtained using the three different procedures was eluted to a final volume of $50 \mu \mathrm{L}$. In summary, these $50 \mu \mathrm{L}$ of eluted DNA derived from $1 / 10$ of the blood spiked with the above number of CFUs (i.e., $10^{6}-10^{0} \mathrm{CFU}$ in each $50 \mu \mathrm{L}$ elution). This implies that the five microliters used as template DNA for real-time PCR contained bacterial DNAs derived from $10^{5}-10^{-1}$ CFU. A series of blood samples spiked as above with E. coli

\begin{tabular}{|c|c|}
\hline Klebsiella pneumoniae & $5^{\prime}-----------------3^{\prime}$ \\
\hline Enterococcus faecalis & $5^{\prime}-----------------3^{\prime}$ \\
\hline Streptococcus pneumoniae & $5^{\prime} \quad----------------\quad 3^{\prime}$ \\
\hline Staphylococcus aureus & $5^{\prime} \quad----------------\quad 3^{\prime}$ \\
\hline Escherichia coli & $5^{\prime}---------------3^{\prime}$ \\
\hline Pseudomonas aeruginosa & $5^{\prime} \quad$------------------ $3^{\prime}$ \\
\hline Acinetobacter baumanii & $5^{\prime}------------------\quad 3^{\prime}$ \\
\hline Candida sp. & $5^{\prime}$ CTAGAGGTGCC-G----- $3^{\prime}$ \\
\hline Homo sapiens & $5^{\prime} \quad$ CA-GAGGTGTC-G----- $3^{\prime}$ \\
\hline Drosophila melanogaster & $5^{\prime}$ CAAGAGGTGTC-G----- $3^{\prime}$ \\
\hline Caenorhabditis elegans & $5^{\prime}$ CA-GAGGTGT-GG----T $3^{\prime}$ \\
\hline PANB-forward & $5^{\prime}$-TCGCTCAACGGATAAA G $3^{\prime}$ \\
\hline
\end{tabular}

\begin{tabular}{|c|c|}
\hline Klebsiella pneumoniae & $5^{\prime} \quad------------------3$ \\
\hline Enterococcus faecalis & $5^{\prime}--------------------3$ \\
\hline Streptococcus pneumoniae & $5^{\prime} \quad---------------------3$ \\
\hline Staphylococcus aureus & $5^{\prime}---------------------3$ \\
\hline Escherichia coli & $5^{\prime}-1-0-1-1$ \\
\hline Pseudomonas aeruginosa & $5^{\prime} \quad---------------------3$ \\
\hline Acinetobacter baumanii & $5^{\prime}----------------------3$ \\
\hline Candida sp. & $5^{\prime} \quad--\mathrm{A}------------\mathrm{A}-\mathrm{AAT} 3$ \\
\hline Homo sapiens & $5^{\prime} \quad--\mathrm{A}-------------\mathrm{A}-\mathrm{AAT} 3$ \\
\hline Drosophila melanogaster & $5^{\prime} \quad--\mathrm{A}-------------\mathrm{A}-\mathrm{AAT} 3$ \\
\hline Caenorhabditis elegans & $5^{\prime} \quad-$ A--------------A-AAT 3 \\
\hline PANB-reverse & $5^{\prime}$ GATGANCCGACATCGAGGTGC 3 \\
\hline
\end{tabular}

Figure 1: Analysis of PANB-forward and PANB-reverse primers homology sequences against the $23 \mathrm{~S}$ rDNA of the most common pathogenic bacteria species, Homo sapiens, Caenorhabditis elegans, Candida albicans and Drosophila melanogaster in their binding areas.

or $S$. aureus were processed for plasma preparation: the spiked series of blood samples were incubated for 2 hours at room temperature (RT) and then centrifuged at $400 \mathrm{~g}$ for 15 minutes at RT. DNA was then extracted as above, using the three different procedures. Each extracted DNA was tested by real-time PCR, and the CT values were applied to the standard curve generated in the same experiment to obtain the corresponding copy number of bacterial $23 \mathrm{~S} \mathrm{rDNA}$ gene targets in each reaction. Additionally, real-time PCR was performed on DNA extracted from blood from healthy donors, used as negative control.

\section{Results}

3.1. Specificity and Sensitivity of the 23S Real-Time PCR Assay. The comparison of the designed primers with the sequences from the 50 bacterial species included in the alignment shows an almost complete identity, with no mismatches at the $3^{\prime}$ end, while a high number of mismatches are present in the alignment with the eukaryotic organisms included, that is, $H$. sapiens, C. elegans, C. albicans, and D. melanogaster, as shown in Figure 1.

In order to validate our in silico findings, the specificity of the novel $23 \mathrm{~S}$ rDNA-targeted primers was also evaluated on a total of 20 Gram-positive and 27 Gramnegative bacterial strains (see Table 1 for details) and on five eukaryotic species, from the genus Candida (see Section 2). PCR amplification was obtained from the 47 bacterial DNAs, 


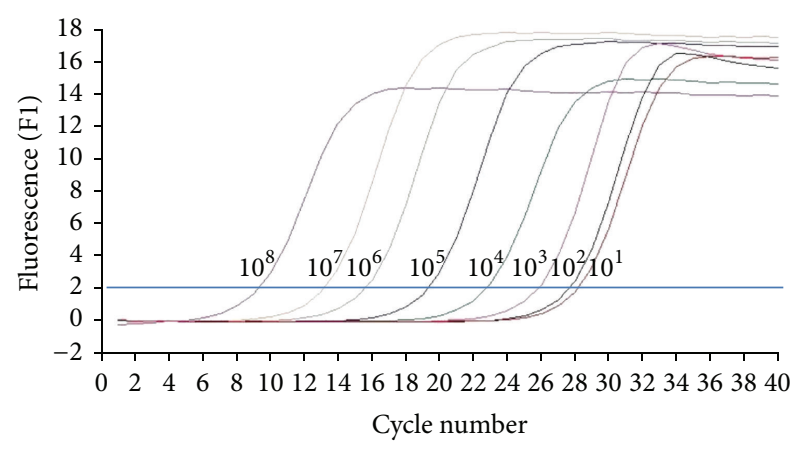

(a)

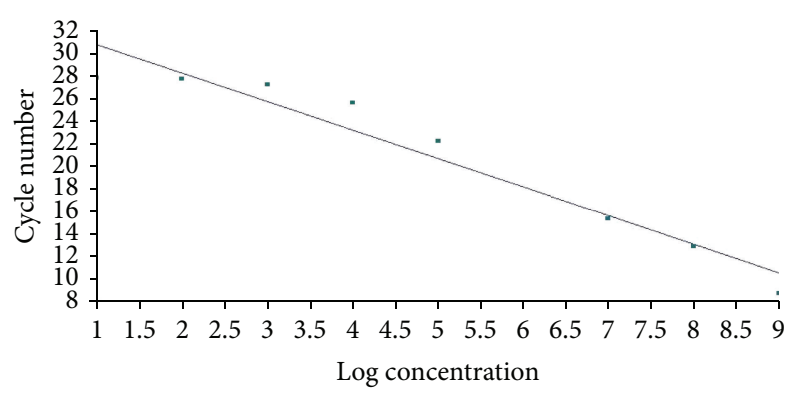

(b)

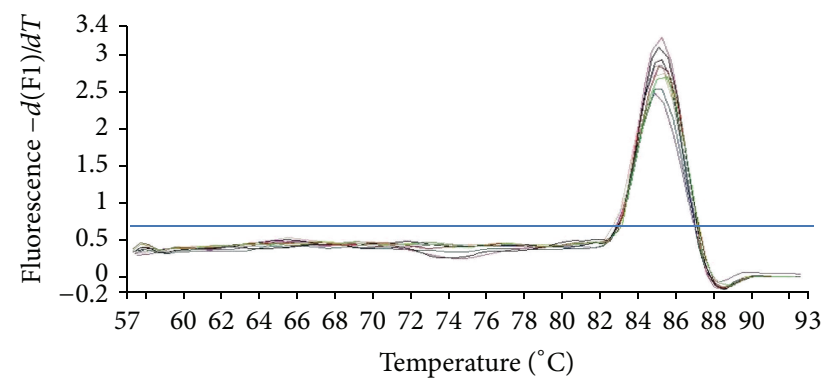

(c)

FIGURE 2: Standard curve amplification of cloned bacterial 23S rDNA plasmid real-time PCR ranging from $10^{8}$ to $10^{1}$ copies per reaction. Panel (a) shows the amplification curve constructed by PCR assay. The fluorescence and the corresponding cycle numbers are shown in the vertical and horizontal axes, respectively. Panel (b) shows the relative standard curve ranging from $10^{1}$ to $10^{8}$ copies per reaction. Panel (c) shows the corresponding melting curve.

with all the melting curves displaying a sharp peak at the expected Tm; no amplification was obtained from any of the Candida-derived DNA (results not shown). Additionally, PCR products obtained from each bacterial amplification were cloned and sequenced as previously described. Sequence of all PCR products obtained was identified as derived from the expected 23S rDNA fragment; these results demonstrate that the developed PCR method specifically detects all the bacterial species tested.

PCR sensitivity was evaluated on a serial dilution of the plasmid containing the 23S rDNA fragment (with each dilution tested in triplicate), at concentrations ranging from $10^{8}$ to $10^{1}$ plasmid copies per reaction, to generate a standard curve ( $R$ value: 0.97 ; slope value: $-2,527$; Figure 2 ). The detection limit of this PCR assay (standard curve method) was 10 copies of $23 \mathrm{~S}$ rDNA copies/reaction. The melting curve analysis of the $23 \mathrm{~S}$ rDNA gene product is shown in Figure 2(c). The electrophoresis run for the PCR products showed a unique specific band of $97 \mathrm{pb}$ corresponding to the $23 \mathrm{~S}$ rDNA gene, thus indicating high specificity. These results demonstrate that the developed PCR method can detect up to 10 copies per reaction, as shown in Figure 2.

3.2. DNA Extraction from Whole Blood and PBS Spiked with Gram-Positive and Gram-Negative Bacteria. We evaluated the efficiency of the two commercially DNA extraction methods in PBS and whole blood spiked with $E$. coli and $S$. aureus. Each experiment included a nonspiked whole blood sample as a negative control. In these negative specimens we never observed a completely negative amplification, given the presence of traces of environmental bacterial DNA. Therefore, in order to set up and define the exact number of $23 \mathrm{~S}$ rDNA copies detected for each sample, the cycle threshold value of the corresponding negative control was used as the edge limit of detection for each run.

The examination of sensitivity for detection of E. coli in PBS after EasyMag extraction showed a positive signal for concentrations in each PCR reaction in the 10 to $10^{2} \mathrm{CFU}$ range; when blood samples were examined, a 10-fold decrease in the detection sensitivity was observed (Figure 3(a)). When $S$. aureus was tested, the detection sensitivity decreased to $10^{3} \mathrm{CFU}$ per PCR in the case of PBS suspension and to above $10^{3} \mathrm{CFU}$ per PCR when whole blood specimens were evaluated, as shown in Figure 3(d).

The QIAmp DNA blood mini kit extraction method showed a detection limit for E. coli of 1 CFU per PCR in PBS and of 10 CFU per PCR in whole blood samples (Figure 3(b)). The lowest detectable concentrations of $S$. aureus in PBS and whole blood specimens were $10^{2}$ and $10^{3}$ CFU per PCR, respectively (Figure $3(\mathrm{e})$ ).

When the pre-treatment step with lysozime and proteinase $\mathrm{K}$ was introduced in the QIAmp extraction protocol the PCR sensitivity rose 10 -fold for $S$. aureus (Figure 3(f)) whereas no increase was demonstrated for $E$. coli (Figure 3(d)). In particular, the detection limit for $E$. coli was 1 and 10 CFU per PCR in PBS and in whole blood, respectively. The detection limit for $S$. aureus $23 \mathrm{~S}$ rDNA ranged from 1 to $10 \mathrm{CFU}$ per PCR and 10 to $10^{2} \mathrm{CFU}$ per PCR, respectively, for the bacterial suspension in PBS and whole blood. Similar results were shown for whole blood samples by Zucol and coworkers by using a set of primers and a specific probe targeted on the 16S RNA gene with an extraction protocol based on enzymatic [6].

\subsection{Comparison of the Bacterial 23S rDNA Copy Number in} Whole Blood and in Plasma. Given the potential application of the novel broad-spectrum PCR assay either in plasma or whole blood specimens, we quantified and compared the detection limit of the novel PCR protocol in whole blood specimens spiked with different bacteria and in the derived corresponding plasma samples, as described above. Our results showed that the EasyMag extraction protocol presented a 100-fold reduction of detection sensitivity in 


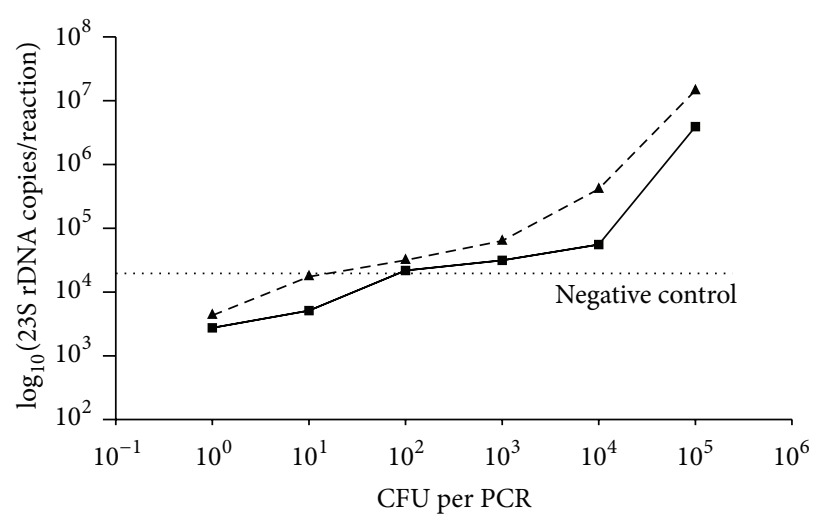

(a)

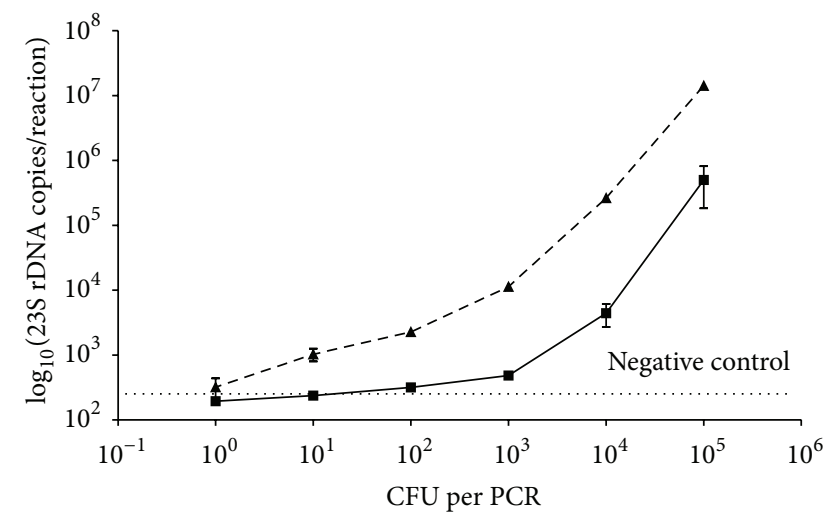

(c)

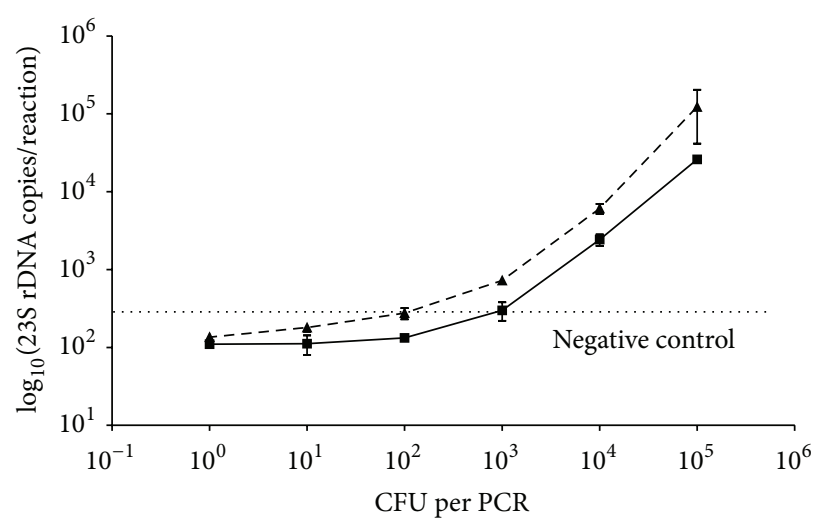

(e)

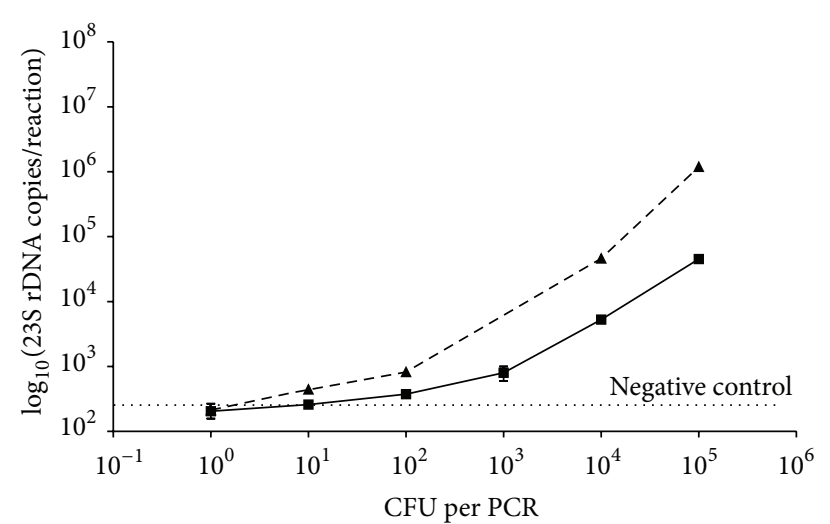

(b)

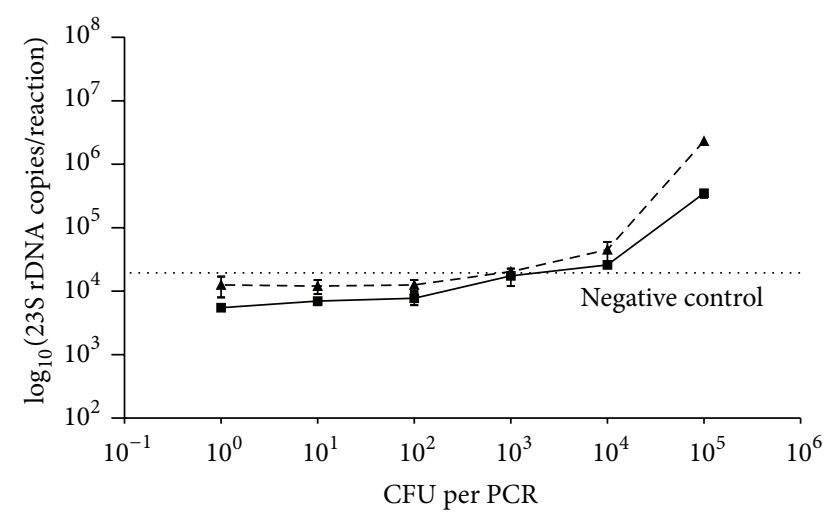

(d)

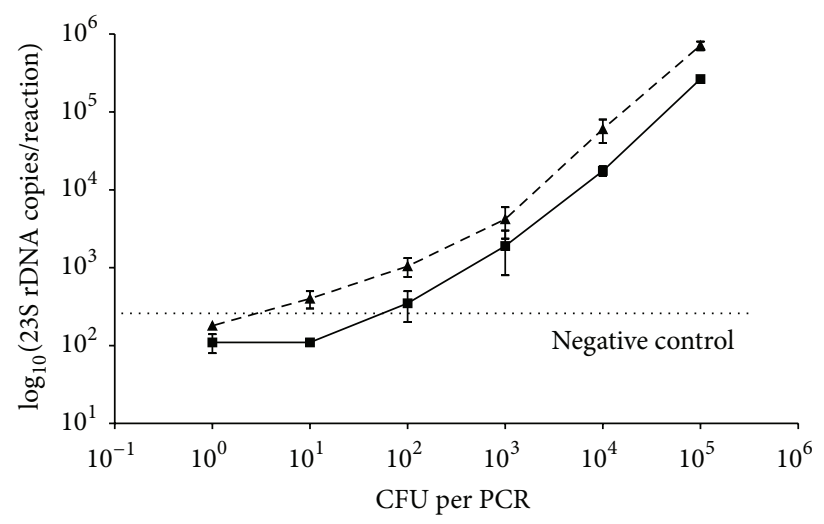

(f)

FIGURE 3: Analytical sensitivities of three different extraction protocols following quantification by the novel 23S rDNA real-time PCR from whole blood (continuous line) and PBS (dotted line) spiked with different Gram-negative (Escherichia coli) or Gram-positive (Staphylococcus aureus) bacteria ranging from 1 to $10^{5}$ bacteria per reaction. EasyMag automated extraction protocols with E. coli (a) or with $S$. aureus (d) spiked in PBS and in whole blood. QIAmp DNA blood mini kit extraction methods with E. coli (b) or with S. aureus (e) spiked in PBS and in whole blood; QIAmp DNA blood mini kit extraction with pretreatment with lysozyme and proteinase K with E. coli (c) or with S. aureus (f), spiked in PBS and in whole blood. Results are representative of three independent experiments, effected on three independent DNA extractions and expressed as mean \pm standard deviations.

the plasma in comparison to the corresponding WB samples (Figures 4(a) and 4(d)). Similarly, the QIAmp DNA blood mini kit with or without the enzymatic pre-treatment showed a similar behavior (Figures 4(b), 4(c), 4(e), and 4(f)). In particular, the QIAmp DNA blood mini kit with pre-treatment showed a positive signal for $E$. coli for as low as $10^{1}$ and $10^{3}$ CFU per PCR from whole blood and plasma, respectively
(Figure 4(c)), and the minimum detection limit for $S$. aureus ranged from $10^{1}$ to $10^{2} \mathrm{CFU}$ per PCR and $10^{2}$ to $10^{3} \mathrm{CFU}$ per PCR from whole blood and plasma (Figure 4(f)). Our results showed that the PCR sensitivity was lower in plasma samples than in the corresponding whole blood samples for both $S$. aureus and E. coli, independently from the extraction method used. 


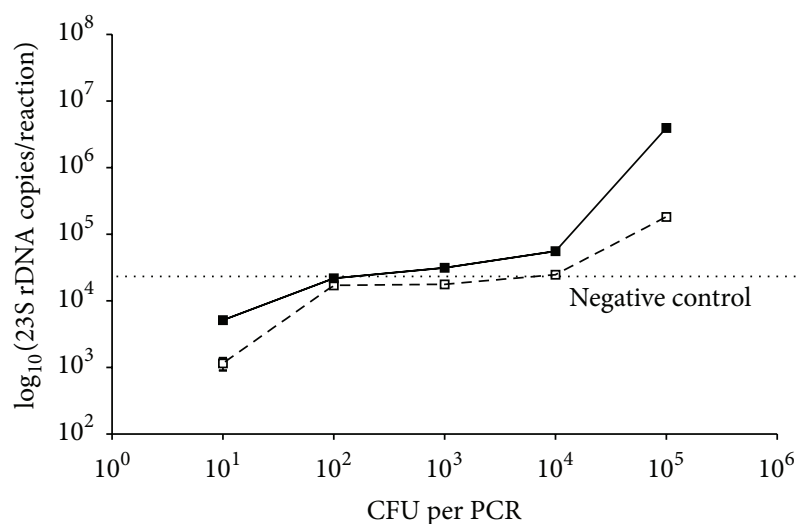

(a)

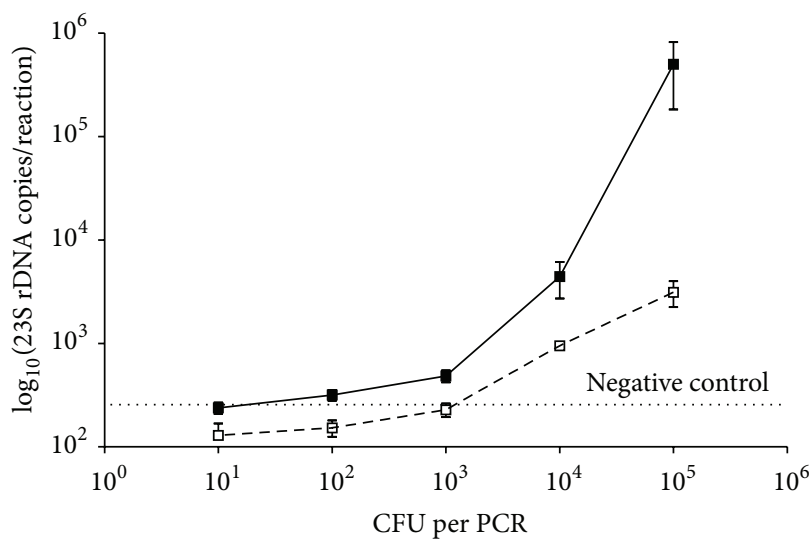

(c)

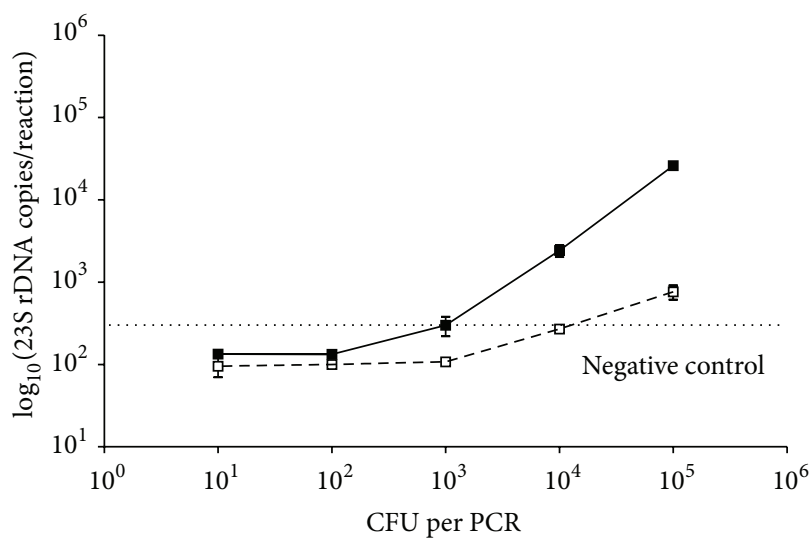

(e)

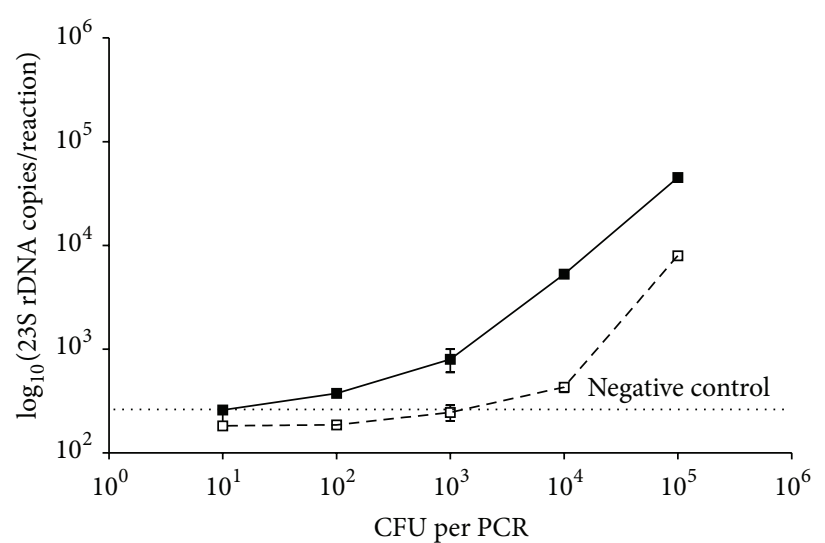

(b)

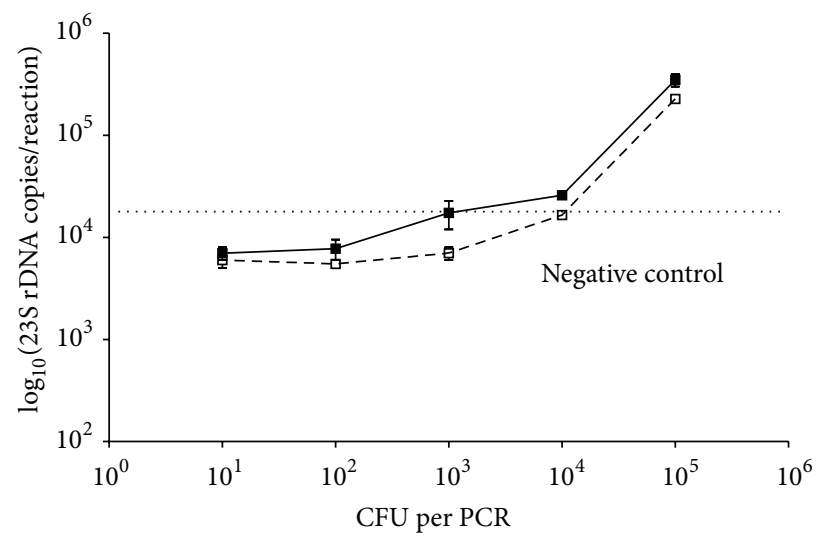

(d)

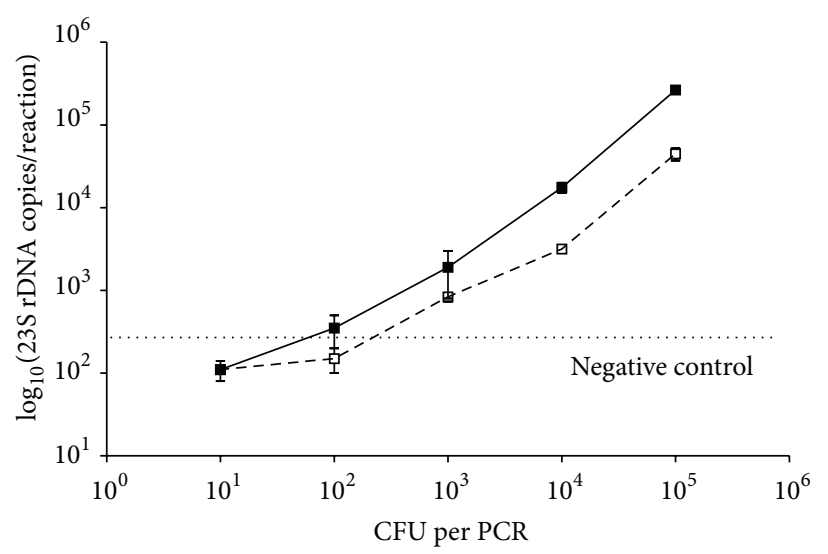

(f)

FIGURE 4: Analytical detection range and sensitivities of different extraction protocols from whole blood (continuous line) spiked with Gramnegative (E. coli) or Gram-positive (S. aureus) bacteria and their corresponding plasma (dotted line). EasyMag protocols with E. coli (a) or with S. aureus (d), spiked in plasma and in whole blood. QIAmp DNA blood mini kit extraction with E. coli (b) or with S. aureus (e), spiked in plasma and in whole blood; QIAmp DNA blood mini kit extraction with pre-treatment with lysozime and proteinase K with $E$. coli (c) or with $S$. aureus (f), in plasma and in whole blood.

\section{Discussion}

In this study, we developed and assessed the analytical performance of a novel real-time PCR method targeted on a conserved region of the $23 \mathrm{~S}$ ribosomal DNA gene, for the detection and quantification of a wide range of human pathogenic bacteria in human blood and plasma samples.
PCR assays capable of detecting a wide range of human pathogens are nowadays important tools in microbiology laboratories, useful for the detection and identification of infecting bacteria, in particular in the case of blood stream infections. This approach demonstrated its utility in cases of patients receiving ongoing antimicrobial therapy or infected by fastidious/not cultivable bacteria $[1,9]$. So far, $16 \mathrm{~S}$ rDNA 
has been the most widely used target for these applications. As detailed in the introduction, one concern that was raised against 16S rDNA-based approaches was its possible lack of specificity, for example, the cross-reactivity with human DNA, but this issue was not largely investigated $[18,23-$ 25]. As target for a pan-bacterial PCR, the $23 \mathrm{~S}$ rDNA region offers some advantages compared to $16 \mathrm{~S}$ rDNA: (i) a higher content of variable sequence stretches, (ii) the presence of unique insertions/deletions, and (iii) possibility of a better phylogenetic resolution because of a higher sequence variation $[19,26,27]$.

The novel 23S rDNA-targeted PCR assay described here was able to specifically detect all of the 47 bacterial isolates included in the study that represent an estimated $90 \%$ of the reported causes of blood stream infections [6]; no false positive reactions were observed when eukaryotic DNAs of diverse origin were tested. A previous study conducted by Zucol and co-workers showed a sensitivity lower than that reported here, when combined nucleic acids extraction methods were applied to water bacterial suspensions and then followed by a $16 \mathrm{SrDNA}$-targeted broad-spectrum real-time PCR assay [6]. As expected, the performance of that method was lower when applied to detect Gram-positive in respect to Gram-negative bacteria, likely due to the thicker cell wall of the former [6].

The minimal detection limits determined for the novel real-time PCR described here were in the range of 1 to $10^{3} \mathrm{CFU}$ per reaction in whole blood spiked with E. coli or S. aureus, depending on DNA extraction methods; as expected, this indicates that the DNA purification step can have a profound effect on the final results. The modified QIAmp DNA blood mini kit, with pre-treatment of the samples with proteinase $\mathrm{K}$ and lysozyme that contribute to the disruption of the bacterial cell envelopes, led to a sensitivity of the overall procedure that appears superior compared to methods without preenzymatic treatments (see Figure 3).

Some previously published studies showed controversial results for the detection of the bacterial $16 \mathrm{~S}$ rDNA in blood. In particular, differences were found when whole blood or plasma specimens were evaluated $[7,15,20,28]$. The effective difference in the amount of bacterial rDNAs detectable in whole blood or plasma samples remains up to today not fully investigated, so that the discussion about the optimal type of samples to be used for the detection of bacterial rDNAs from blood stream specimens is still ongoing [2]. In this study, we tested whole blood samples spiked with either $E$. coli or $S$. aureus and the derived plasma specimens, demonstrating that the detection of $23 \mathrm{~S}$ rDNA was more effective when whole blood was used.

These results suggest that for an appropriate and effective detection of bacterial 23S rDNA the most promising type of sample is whole blood, while plasma must be considered a second choice, given the lower detection limit. A major weakness of the novel test described in this paper is the short sequence, 97 base pair, used as target for the assay, that makes quite unlikely the possibility to use the amplicons for a successive species identification, based on sequence analysis.
On the other hand, it should be considered that in most cases of invasive bacterial disease and in particular in blood stream infections, the bacterial load in biological samples, such as cerebrospinal fluid and blood, is usually quite low and the use of a very sensitive diagnostic method, like the novel PCR assay described in this paper, is highly desirable in order to prove the microbial etiology. A similar approach was recently described by Banada and co-workers, who showed that an increase in the sensitivity of the PCR protocol can be obtained by a decrease in amplicon size [28]. Additional methods, capable of identifying the infective germs could be applied later on in the diagnostic workflow, eventually with additional steps capable of increasing the bacterial load, such as enrichment broth culture or testing on specimens obtained after withdrawal or suspension of the antimicrobial therapy. The presence of bacterial products, including nucleic acids, in the blood is nowadays a well-recognized phenomenon that frequently occurs when pathological conditions allow translocation from highly colonized sites, such as the bowel $[29,30]$. One major application of the method in this study could be the quantification of total bacterial DNA in the bloodstream in all the cases in which a sensitive detection of DNA is desired without the need for a precise bacterial species identification.

\section{Authors' Contribution}

P. Gaibani and M. Mariconti equally contributed to this paper.

\section{Acknowledgments}

The authors thank M. Cordovana for the selection of the BACSO isolates utilized in this study. They thank Chiara Bazzocchi for useful discussion. This study was supported by funds RFO 2010 and 2011 from University of Bologna to V. Sambri and by funds IRCCS Policlinico S. Matteo Pavia to S. Novati.

\section{References}

[1] J. M. Mylotte and A. Tayara, "Blood cultures: clinical aspects and controversies," European Journal of Clinical Microbiology and Infectious Diseases, vol. 19, no. 3, pp. 157-163, 2000.

[2] M. Paolucci, M. P. Landini, and V. Sambri, "Conventional and molecular techniques for the early diagnosis of bacteraemia," International Journal of Antimicrobial Agents, vol. 36, no. 2, pp. S6-S16, 2010.

[3] P. Gaibani, G. Rossini, S. Ambretti et al., "Blood culture systems: rapid detection: how and why?" International journal of antimicrobial agents, vol. 34, pp. S13-S15, 2009.

[4] R. M. Anthony, T. J. Brown, and G. L. French, "Rapid diagnosis of bacteremia by universal amplification of $23 \mathrm{~S}$ ribosomal DNA followed by hybridization to an oligonucleotide array," Journal of Clinical Microbiology, vol. 38, no. 2, pp. 781-788, 2000.

[5] A. L. Rosey, E. Abachin, G. Quesnes et al., "Development of a broad-range $16 \mathrm{~S}$ rDNA real-time PCR for the diagnosis of septic arthritis in children," Journal of Microbiological Methods, vol. 68, no. 1, pp. 88-93, 2007. 
[6] F. Zucol, R. A. Ammann, C. Berger et al., "Real-time quantitative broad-range PCR assay for detection of the 16S rRNA gene followed by sequencing for species identification," Journal of Clinical Microbiology, vol. 44, no. 8, pp. 2750-2759, 2006.

[7] W. Jiang, M. M. Lederman, and P. Hunt, "Plasma levels of bacterial DNA correlate with immune activation and the magnitude of immune restoration in persons with antiretroviral-treated HIV infection," Journal of Infectious Diseases, vol. 199, no. 1177, p. 1185, 2009.

[8] K. Matsuda, H. Tsuji, T. Asahara, Y. Kado, and K. Nomoto, "sensitive quantitative detection of commensal bacteria by rRNA-targeted reverse transcription-PCR," Applied and Environmental Microbiology, vol. 73, no. 1, pp. 32-39, 2007.

[9] S. K. Rampini, G. V. Bloemberg, P. M. Keller et al., "Broadrange $16 \mathrm{~S}$ rRNA gene polymerase chain reaction for diagnosis of culture-negative bacterial infections," Clinical Infectious Diseases, vol. 53, no. 12, pp. 1245-1251, 2011.

[10] S. Yang, S. Lin, G. D. Kelen et al., "Quantitative multiprobe PCR assay for simultaneous detection and identification to species level of bacterial pathogens," Journal of Clinical Microbiology, vol. 40, no. 9, pp. 3449-3454, 2002.

[11] P. Zapater, R. Francés, J. M. González-Navajas et al., "Serum and ascitic fluid bacterial DNA: a new independent prognostic factor in noninfected patients with cirrhosis," Hepatology, vol. 48, no. 6, pp. 1924-1931, 2008.

[12] J. E. Clarridge, "Impact of $16 \mathrm{~S}$ rRNA gene sequence analysis for identification of bacteria on clinical microbiology and infectious diseases," Clinical Microbiology Reviews, vol. 17, no. 4, pp. 840-862, 2004.

[13] T. Bacchetti De Gregoris, N. Aldred, A. S. Clare, and J. G. Burgess, "Improvement of phylum- and class-specific primers for real-time PCR quantification of bacterial taxa," Journal of Microbiological Methods, vol. 86, no. 3, pp. 351-356, 2011.

[14] A. Cherkaoui, S. Emonet, D. Ceroni et al., "Development and validation of a modified broad-range 16S rDNA PCR for diagnostic purposes in clinical microbiology," Journal of Microbiological Methods, vol. 79, no. 2, pp. 227-231, 2009.

[15] E. Ferri, S. Novati, M. Casiraghi et al., "Plasma levels of bacterial DNA in HIV infection: the limits of quantitative polymerase chain reaction," Journal of Infectious Diseases, vol. 202, no. 1, pp. 176-177, 2010.

[16] V. Gentili, P. G. Balboni, E. Menegatti et al., "Panbacterial real-time PCR to evaluate bacterial burden in chronic wounds treated with Cutimed Sorbact," European Journal of Clinical Microbiology and Infectious Diseases, vol. 31, no. 7, pp. 1523-1529, 2011.

[17] E. T. Zemanick, B. D. Wagner, S. D. Sagel, M. J. Stevens, F. J. Accurso, and J. Kirk Harris, "Reliability of quantitative real-time PCR for bacterial detection in cystic fibrosis airway specimens," PLoS ONE, vol. 5, no. 11, Article ID e15101, 2010.

[18] Ø. Kommedal, K. Simmon, D. Karaca, N. Langeland, and H. G. Wikera, "Dual priming oligonucleotides for broad-range amplification of the bacterial 16S rRNA gene directly from human clinical specimens," Journal of Clinical Microbiology, vol. 50, no. 4, pp. 1289-1294, 2012.

[19] D. E. Hunt, V. Klepac-Ceraj, S. G. Acinas, C. Gautier, S. Bertilsson, and M. F. Polz, "Evaluation of $23 \mathrm{~S}$ rRNA PCR primers for use in phylogenetic studies of bacterial diversity," Applied and Environmental Microbiology, vol. 72, no. 3, pp. 22212225,2006

[20] S. Nikkari, I. J. McLaughlin, W. Bi, D. E. Dodge, and D. A. Relman, "Does blood of healthy subjects contain bacterial ribosomal DNA?" Journal of Clinical Microbiology, vol. 39, no. 5, pp. 1956-1959, 2001.

[21] R. C. Edgar, "MUSCLE: multiple sequence alignment with high accuracy and high throughput," Nucleic Acids Research, vol. 32, no. 5, pp. 1792-1797, 2004.

[22] S. Epis, P. Gaibani, U. Ulissi, B. Chouaia, I. Ricci et al., "Do mosquito-associated bacteria of the genus Asaia circulate in humans?" European Journal of Clinical Microbiology \& Infectious Diseases, vol. 31, pp. 1137-1140, 2012.

[23] K. A. Harris and J. C. Hartley, "Development of broad-range $16 S$ rDNA PCR for use in the routine diagnostic clinical microbiology service," Journal of Medical Microbiology, vol. 52, no. 8, pp. 685-691, 2003.

[24] K. Rantakokko-Jalava, S. Nikkari, J. Jalava et al., "Direct amplification of rRNA genes in diagnosis of bacterial infections," Journal of Clinical Microbiology, vol. 38, no. 1, pp. 32-39, 2000.

[25] B. Vandercam, S. Jeumont, O. Cornu et al., "Amplification-based DNA analysis in the diagnosis of prosthetic joint infection," Journal of Molecular Diagnostics, vol. 10, no. 6, pp. 537-543, 2008.

[26] W. Ludwig and K. H. Schleifer, "Bacterial phylogeny based on $16 \mathrm{~S}$ and $23 \mathrm{~S}$ rRNA sequence analysis," FEMS Microbiology Reviews, vol. 15, no. 2-3, pp. 155-173, 1994.

[27] A. Pei, C. W. Nossa, P. Chokshi et al., "Diversity of $23 S$ rRNA genes within individual prokaryotic genomes," PloS One, vol. 4, no. 5, Article ID e5437, 2009.

[28] P. P. Banada, S. Chakravorty, D. Shah, M. Burday, F. M. Mazzella, and D. Alland, "Highly sensitive detection of staphylococcus aureus directly from patient blood," PLoS ONE, vol. 7, no. 2, Article ID e31126, 2012.

[29] I. Gómez-Hurtado, A. Santacruz, G. Peiró et al., "Gut microbiota dysbiosis is associated with inflammation and bacterial translocation in mice with CCl4-Induced fibrosis," PLOS ONE, vol. 6, no. 7, Article ID e23037, 2011.

[30] M. Kramski, A. J. Gaeguta, R. Rajasuriar et al., "Novel sensitive real-time PCR for quantification of bacterial 16S rRNA genes in plasma of HIV-infected patients as a marker for microbial translocation," Journal of Clinical Microbiology, vol. 49, no. 10, pp. 3691-3693, 2011. 

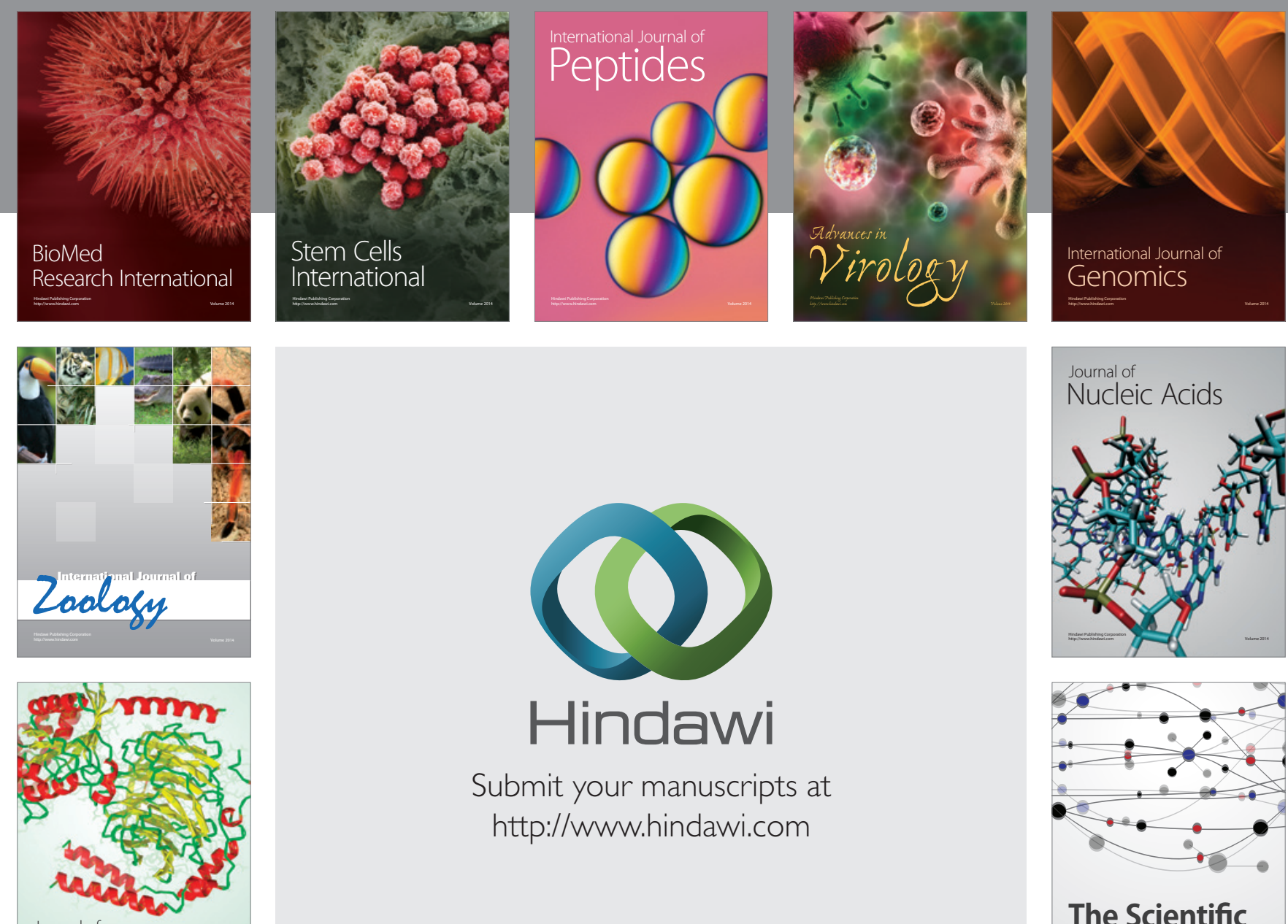

Submit your manuscripts at

http://www.hindawi.com

Journal of
Signal Transduction
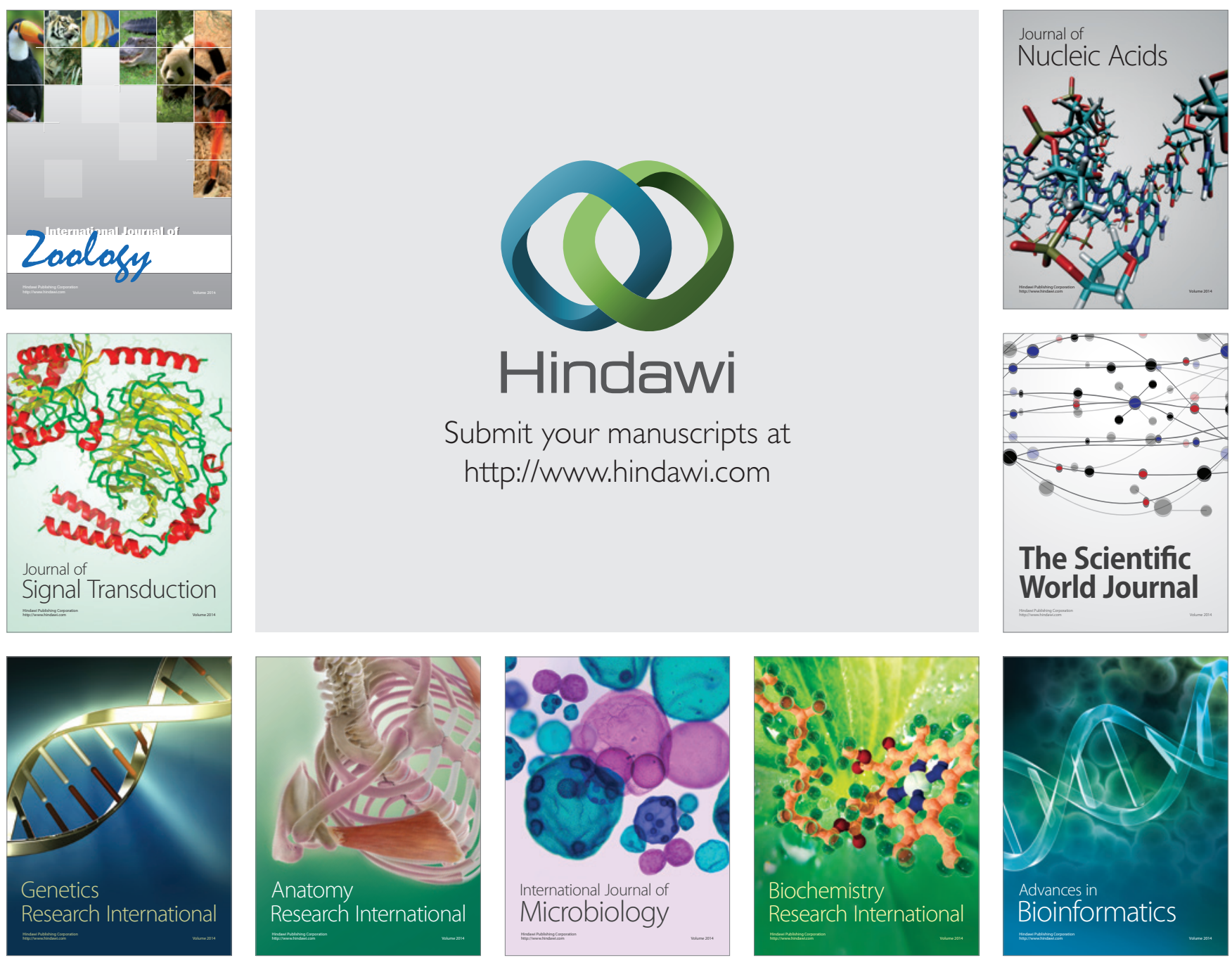

The Scientific World Journal
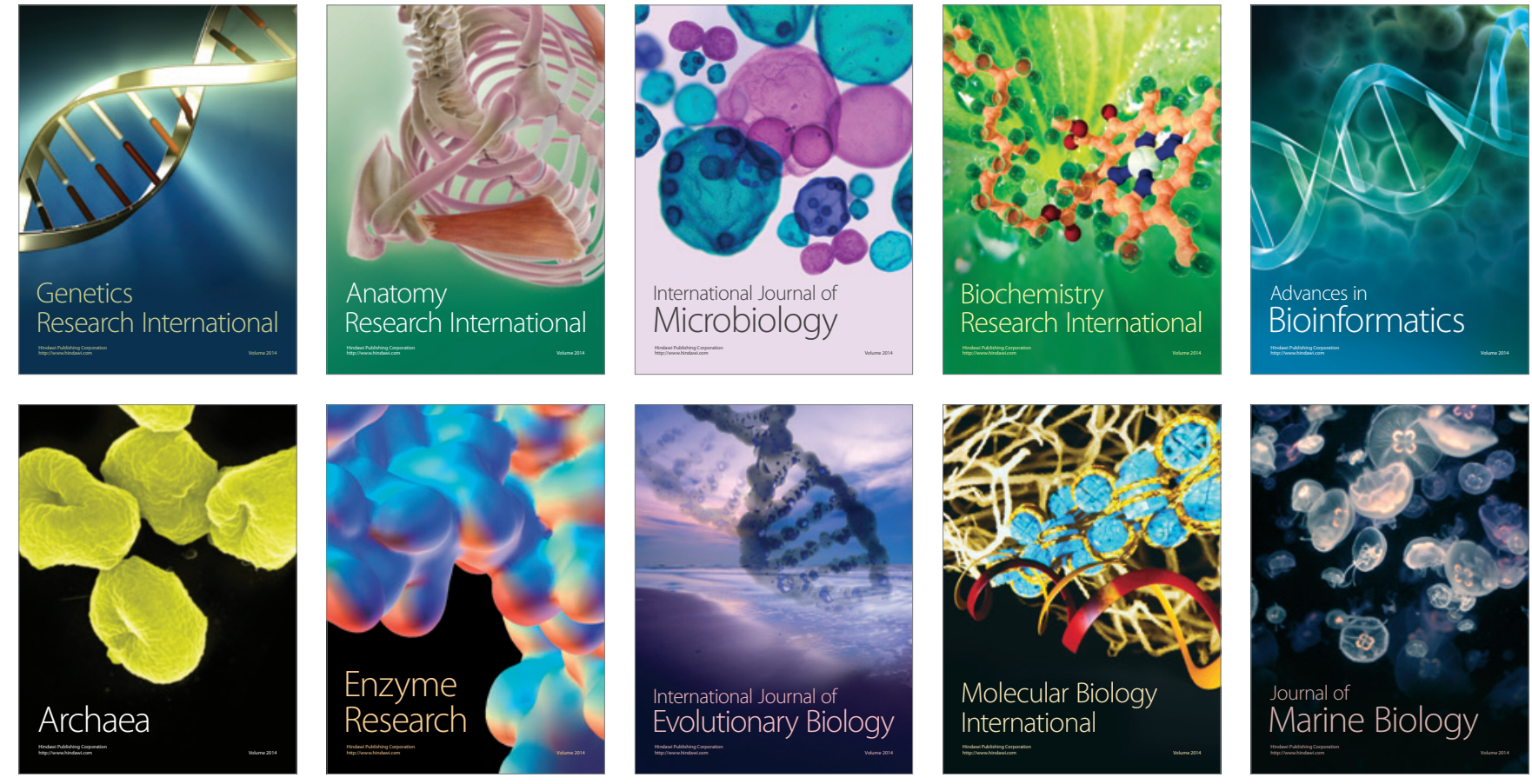